\title{
Volatile $p$-Nitroaniline as Matrix for High Spatial Resolution Imaging of Phospholipids in Both Ion Modes by AP-MALDI-IMS
}

\author{
Shoko Matsushita, Eiji Sugiyama, Takahiro Hayasaka, Noritaka Masaki, Mitsutoshi Setou
}

Department of Cell Biology and Anatomy, Hamamatsu University School of Medicine, Hamamatsu, Japan

Matrix-assisted laser desorption/ionization imaging mass spectrometry (MALDI-IMS) is a useful techniques that allows to derive the spatial distribution of numerous molecules ionized on a tissue section. Selection of the suitable matrix is the key to ionize molecules of interest in MALDI. Each matrix has its own characteristics such as being proper for ionizing molecules in either positive or negative ion mode, different crystallization, suitability for specific biomolecules, and so on. 2,5-dihydroxybenzoic acid (DHB) and 9-aminoacridine (9AA) are conventionally used as matrices in MALDI. These matrices are used for particular ion mode in MALDI-IMS, DHB for positive and 9AA for negative ion mode. Therefore, we prepared two serial sections to reveal the distribution of biomolecules in both ion modes. It is desirable to find a new matrix which is suitable for general purpose, for example is applicable in both ion modes. Such a matrix can reduce the loss of rare or hard to obtain samples, such as clinical ones. Recently, volatile materials gathered interest as MALDI matrices. Although volatile matrices are expected to be useful for detecting new molecules, they are unstable in vacuum condition. Here, we utilized a volatile matrix for atmospheric pressure (AP)-MALDI-IMS instrument, Mass Microscope (Shimadzu). The instrument had been developed in our laboratory in collaboration with Shimadzu. We selected $p$-nitroaniline (NIT) as a volatile matrix that is expected to be applicable for IMS in both ion modes because previous reports show that NIT can be used for MALDI-MS analysis in both ion modes. In this study, we optimized the pretreatment using NIT in order to perform high spatial resolution ion imaging in both ion modes by using Mass Microscope.

We optimized the pretreatment using NIT, as it is the first application of this matrix for AP-MALDI-IMS. As samples, mouse liver and brain were analyzed. Fresh frozen tissues were sectioned at thickness of $10 \mathrm{~mm}$ using CM1950 cryostat (Leica). Frozen sections were thaw-mounted on conductive glass slides coated with indium-tin-oxide. And then, NIT was manually sprayed upon the slide glass using air-brush. To maximize signals, different working volumes and solvent amounts were tested. We also compared the signals obtained using NIT to signals obtained using conventional matrices, DHB and 9AA. Using optimized pretreatment, MS/MS analysis and high resolution imaging were demonstrated. For this purpose, the diameter of laser spot was fixed at $5 \mu \mathrm{m}$ in this study.

By optimizing the pretreatment using NIT matrix, we could obtain the mass spectrum as well or better than when using DHB and 9AA matrices for the mass range from $\mathrm{m} / \mathrm{z} 700$ to 900 in mouse liver, where signals corresponding to lipids are concentrated. The most intense signal detected in positive ion mode was identified as phosphatidylcholine (PC) using MS/MS analysis. Similarly, two of the most intense signals detected in negative ion mode were identified as phosphatidylethanolamine (PE) and phosphatidylinositol (PI), respectively. Using this optimized condition, improvement of signals intensity enables us to visualize the distribution with high spatial resolution imaging of $5 \mu \mathrm{m}$ in both ion modes, even for adjacent region on the same mouse brain cerebellum section (Figure). 
As demonstrated in this study, combined usage of NIT with AP-MALDI-IMS allows to analyze biological samples both in good sensitivity and high spatial resolution, and in addition, requires only one time sample pretreatment. Utilization of NIT is especially powerful for phospholipid analysis as it can visualize phospholipid distribution at high spatial resolution of $5 \mu \mathrm{m}$ and is also available for the identification of phospholipid species by MS/MS analysis. It is expected that more information from rare samples can be extracted using the combination of NIT and AP-MALDI-IMS.

We could visualize phospholipids with high spatial resolution in both ion modes by AP-MALDI-IMS using a volatile matrix, $p$-nitroaniline.

\section{References}

[1] A. Thomas et al, Analytical Chemistry 84 (2012), p. 2048.

[2] R. T. Steven, A. M. Race and J. Bunch, Journal of The American Society for Mass Spectrometry 24 (2013), p. 801.

[3] R. T. Steven, J. Bunch, Analytical and Bioanalytical Chemistry 405 (2013), p. 4719.

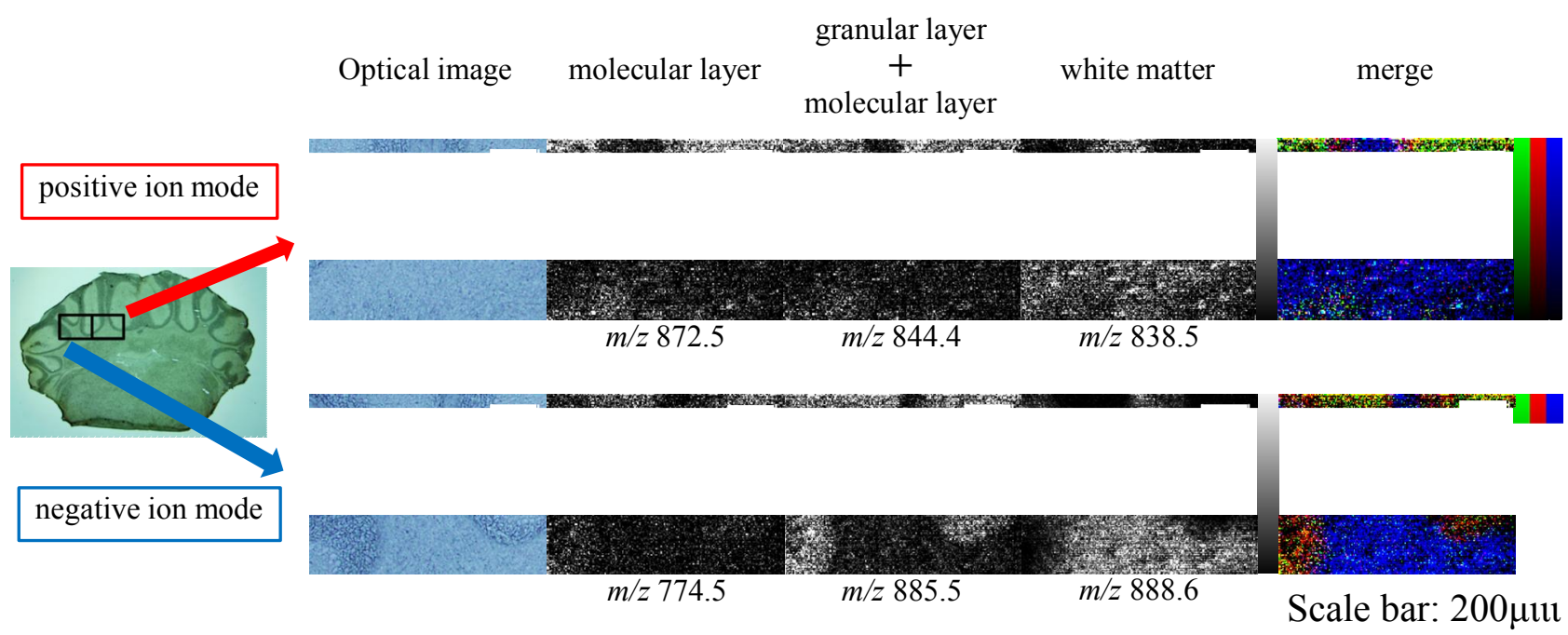

High spatial resolution imaging of a mouse cerebellum in positive and negative ion mode. The optical image of the whole mouse brain coronal section analyzed in this study is shown in the left hand panel. The enlarged optical image analyzed in positive ion mode is shown in upper part of the figure, together with representative ion images shown in 8-bit gray scale and their merged color image. Similarly, the result of negative ion mode measurement is shown in lower part of the figure. Representative ion images associated with anatomical structures are shown. The merged images in both ion modes clearly distinguish different molecular compositions among these layers and depict anatomical structures even about the tiny distribution of granular layer within $50 \mu \mathrm{m}$. The colors in merged images are shown using pseudocolor scales; green represents molecular layer, red represents both granular layer and molecular layer, and blue represents white matter. 